\title{
Correction to: Implementation, efficacy, costs and processes of inpatient equivalent hometreatment in German mental health care (AKtiV): protocol of a mixed-method, participatory, quasi-experimental trial
}

\author{
Johanna Baumgardt ${ }^{{ }^{*}+}$, Julian Schwarz ${ }^{2+}$, Andreas Bechdolf ${ }^{1,3,4}$, Konstantinos Nikolaidis ${ }^{1}$, Martin Heinze ${ }^{2}$, \\ Johannes Hamann ${ }^{5,6}$, Martin Holzke ${ }^{7}$, Gerhard Längle ${ }^{8,9,10}$, Janina Richter ${ }^{10}$, Peter Brieger ${ }^{5}$, Reinhold Kilian ${ }^{11}$, \\ Jürgen Timm ${ }^{12}$, Constance Hirschmeier ${ }^{13}$, Sebastian Von Peter ${ }^{2+}$ and Stefan Weinmann ${ }^{14,15 \dagger}$
}

Correction to: BMC Psychiatry 21, 173 (2021)
https://doi.org/10.1186/s12888-021-03163-9

Following the publication of the original article [1], the authors identified errors in the authors names. The given names and family names were erroneously transposed.

The incorrect authors names are:

Baumgardt Johanna1* + , Schwarz Julian2†, Bechdolf Andreas1,3,4, Nikolaidis Konstantinos1, Heinze Martin2, Hamann Johannes5,6, Holzke Martin7, Längle Gerhard8,9,10, Richter Janina10, Brieger Peter5, Kilian Reinhold11, Timm Jürgen12, Hirschmeier Constance13, Von Peter Sebastian2† and Weinmann Stefan14,15†

The correct authors names are:

Johanna Baumgardt1* + , Julian Schwarz2+, Andreas Bechdolf1,3,4, Konstantinos Nikolaidis1, Martin

The original article can be found online at https://doi.org/10.1186/s12888021-03163-9.

* Correspondence: johanna.baumgardt@vivantes.de

${ }^{\dagger}$ Johanna Baumgardt, Julian Schwarz, Sebastian Von Peter and Stefan Weinmann contributed equally to this work.

'Department of Psychiatry, Psychotherapy and Psychosomatic Medicine,

Vivantes Hospital Am Urban und Vivantes Hospital im Friedrichshain, Charité

- Universitätsmedizin Berlin, Vivantes Klinikum Am Urban, Berlin, Germany

Full list of author information is available at the end of the article
Heinze2, Johannes Hamann5,6, Martin Holzke7, Gerhard Längle8,9,10, Janina Richter10, Peter Brieger5, Reinhold Kilian11, Jürgen Timm12, Constance Hirschmeier13, Sebastian Von Peter2 $\dagger$ and Stefan Weinmann14,15+

The author group has been updated above and the original article [1] has been corrected.

\section{Author details}

'Department of Psychiatry, Psychotherapy and Psychosomatic Medicine, Vivantes Hospital Am Urban und Vivantes Hospital im Friedrichshain, Charité - Universitätsmedizin Berlin, Vivantes Klinikum Am Urban, Berlin, Germany.

${ }^{2}$ Department of Psychiatry and Psychotherapy, Brandenburg Medical School Theodor Fontane, Immanuel Clinic Rüdersdorf, Rüdersdorf, Germany. ${ }^{3}$ ORYGEN, National Center of Excellence of Youth Mental Health, University of Melbourne, Melbourne, Australia. ${ }^{4}$ Department for Psychiatry and Psychotherapy, University Hospital Cologne, Cologne, Germany. ${ }^{5}$ kbo-Isar Amper Klinikum, Region München, Munich, Germany. ${ }^{6}$ Department of Psychiatry and Psychotherapy, Technical University of Munich, Munich, Germany. ${ }^{7}$ Center for Psychiatry Suedwuerttemberg, Department of Psychiatry I, Ulm University, Ravensburg, Weissenau, Germany. ${ }^{8}$ Center for Psychiatry Suedwuerttemberg, Zwiefalten, Germany. ${ }^{9}$ Gemeinnützige $\mathrm{GmbH}$ für Psychiatrie Reutlingen (PP.rt), Academic Hospital of Tuebingen University, Reutlingen, Germany. ${ }^{10}$ Department of Psychiatry and Psychotherapy, University Hospital Tuebingen, Department of Medicine of the Tuebingen University, Tuebingen, Germany. ${ }^{11}$ Department of Psychiatry II, UIm University, Günzburg, Germany. ${ }^{12}$ University of Bremen, Bremen, Germany. ${ }^{13}$ Department for Psychiatry and Psychotherapy, Charité University Hospital Berlin, Berlin, Germany. ${ }^{14}$ Psychiatric Hospital and Rehabilitation Unit,

C The Author(s). 2021 Open Access This article is licensed under a Creative Commons Attribution 4.0 International License, which permits use, sharing, adaptation, distribution and reproduction in any medium or format, as long as you give appropriate credit to the original author(s) and the source, provide a link to the Creative Commons licence, and indicate if changes were made. The images or other third party material in this article are included in the article's Creative Commons licence, unless indicated otherwise in a credit line to the material. If material is not included in the article's Creative Commons licence and your intended use is not permitted by statutory regulation or exceeds the permitted use, you will need to obtain permission directly from the copyright holder. To view a copy of this licence, visit http://creativecommons.org/licenses/by/4.0/ The Creative Commons Public Domain Dedication waiver (http://creativecommons.org/publicdomain/zero/1.0/) applies to the data made available in this article, unless otherwise stated in a credit line to the data. 
Rudolf-Sophien-Stift, Stuttgart, Germany. ${ }^{15}$ University Psychiatric Hospital Basel, Basel, Switzerland.

Published online: 19 April 2021

Reference

1. Baumgardt J, et al. Implementation, efficacy, costs and processes of inpatient equivalent hometreatment in German mental health care (AKtiV): protocol of a mixed-method, participatory, quasi-experimental trial. BMC Psychiatry. 2021;21:173. https://doi.org/10.1186/s12888-021-03163-9. 